\title{
Multiple regulators contribute to the transcriptional control of the Candidalysin-encoding gene ECE1
}

Ronny Martin1, Sophia Ruben², Enrico Garbe ${ }^{3}$ and Oliver Kurzai ${ }^{1,2,4}$

${ }^{1}$ Institute for Hygiene and Microbiology, University of Würzburg, Würzburg, Germany

2Septomics Research Center, Leibniz Institute for Natural Product Research and Infection Biology- Hans Knoell Institute and Friedrich Schiller University, Jena, Germany

${ }^{3}$ Research Group Host-Fungal Interfaces, Leibniz Institute for Natural Product Research and Infection Biology- Hans Knoell Institute and Friedrich Schiller University, Jena, Germany

${ }^{4}$ National Reference Center for Invasive Fungal Infections, Leibniz Institute for Natural Product Research and Infection Biology- Hans Knoell Institute, Jena, Germany

\section{Introduction}

Candidalysin is a toxic peptide which is secreted by $C$. albicans hyphae during the infection of human host cells. The peptide is derived from a precursor protein which is encoded by the ECE1 gene. ECE1 is normally the most abundant transcript in hyphae, but it is not expressed in yeast cells. The aim of this study was to identify regulators of ECE1 transcription.

\section{Visualization of ECE1 transcription}

To establish an ECE1-GFP reporter system (pECE1-GFP), one ECE1 allele was replaced by GFP. The strain was then grown for $6 \mathrm{~h}$ at $37^{\circ} \mathrm{C}$ in either SDG or SDG with $10 \%$ human serum (HS). Only hyphae showed a GFP signal. This observation correlates with the qRT PCR results.
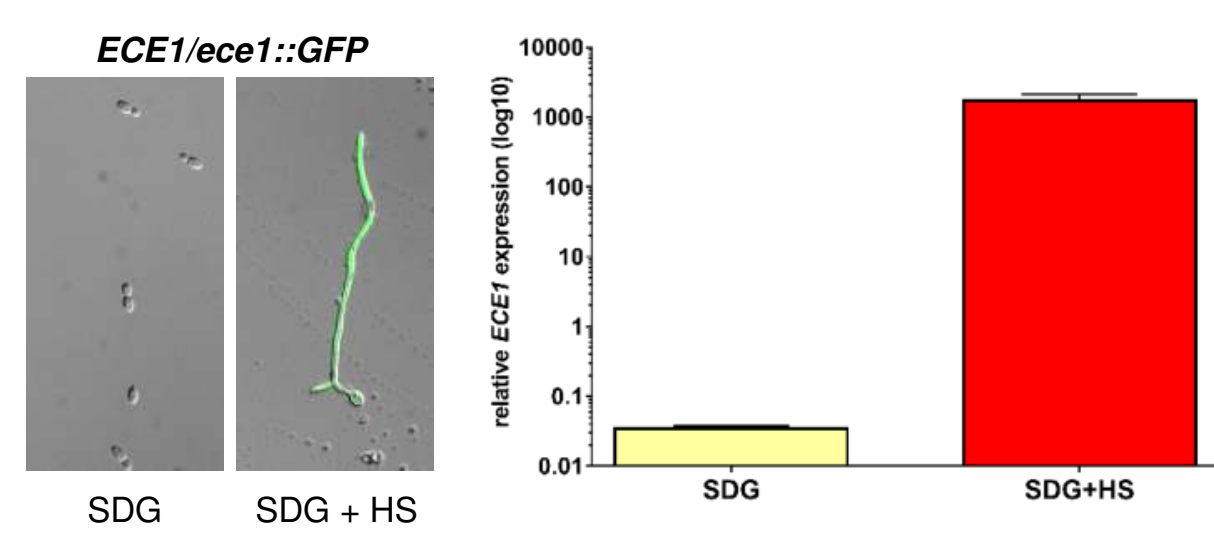

Transcriptional regulators Ahr1 and Tup1 are required for high level expression of ECE1

pECE1-GFP in activator mutants

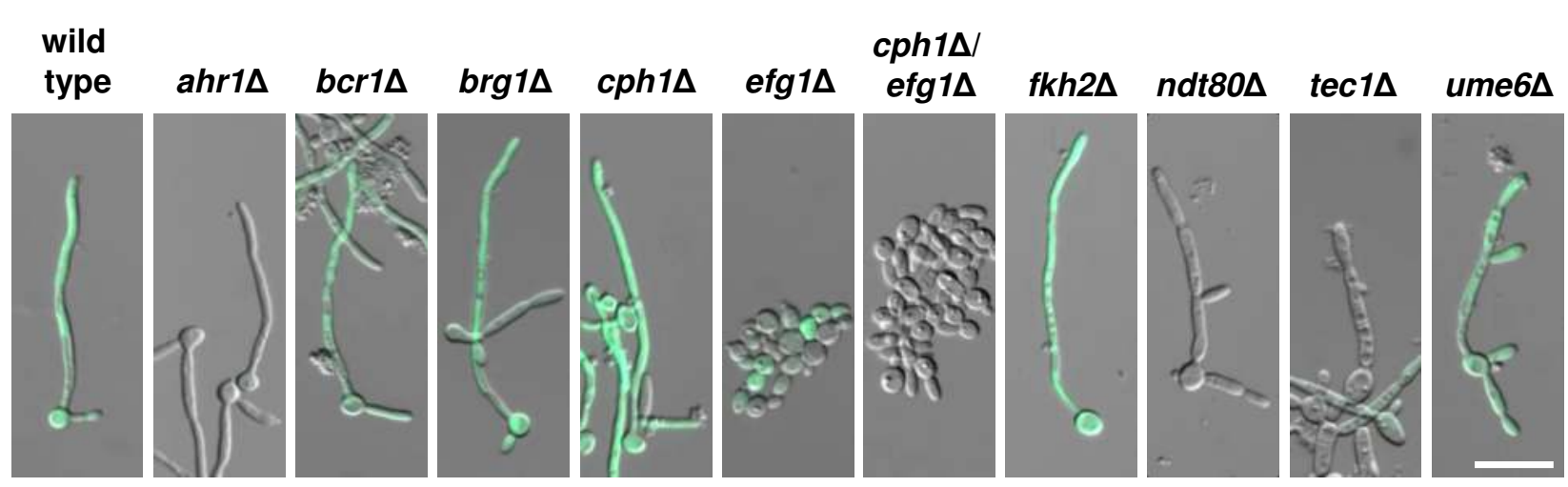

The indicated strains were grown in $\mathrm{SDG}+\mathrm{HS}$ for $6 \mathrm{~h}$ at $37^{\circ} \mathrm{C}$ prior to microscopy. The repressor mutants were also incubated in SDG $\left(6 \mathrm{~h}, 37^{\circ} \mathrm{C}\right)$. Scale bar: $20 \mu \mathrm{m}$.
pECE1-GFP in repressor mutants

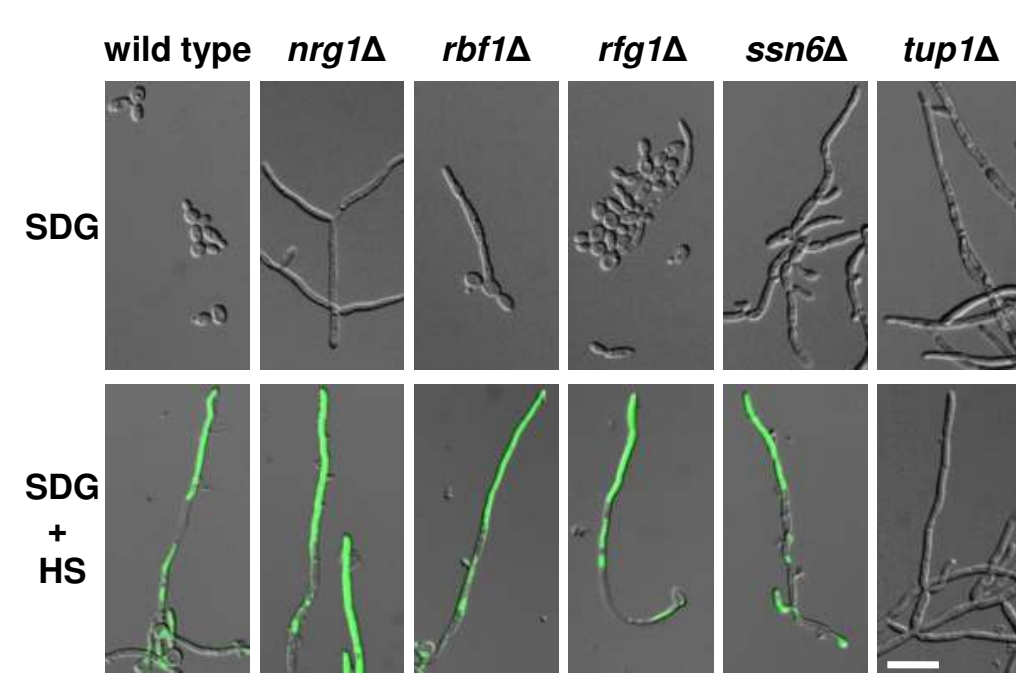

Low ECE1 expression in

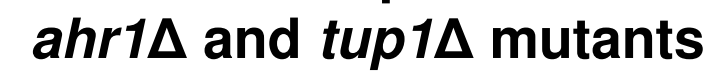

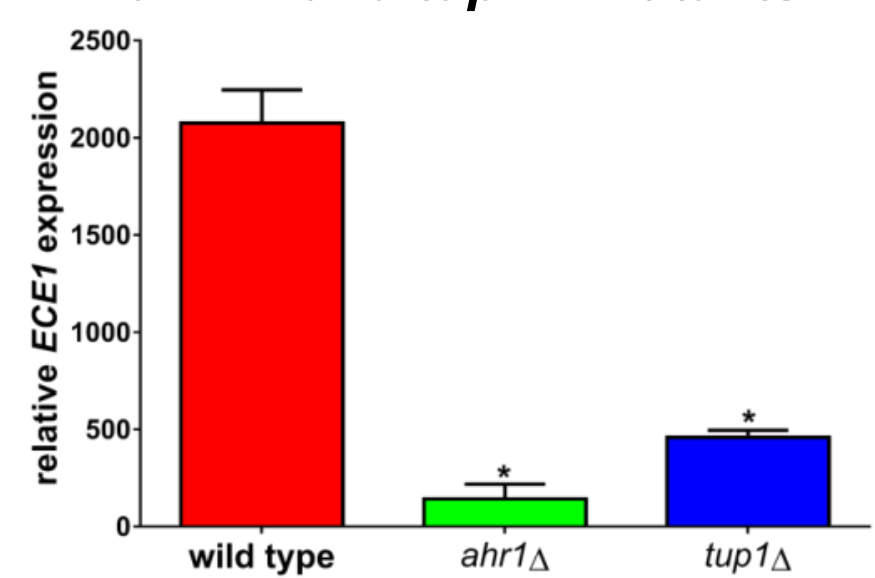

RNA was isolated after 6 h growth in SDG+HS at $37^{\circ} \mathrm{C}$. Gene expression was normalized against ACT1 and a control RNA (5h YPD, $\left.37^{\circ} \mathrm{C}\right)$. Asterisks mark significant differences $(\mathrm{p}$ $\leq 0.05$, two-tailed, unpaired student's $t$-test).

Stimulus-independent activation of ECE1 by a hyperactive $A H R 1$ allele

A hyperactive $A H R 1$ allele ( $A H R 1-G A D)$ triggers expression of ECE1 without additional stimuli. This up-regulation can also be observed in various regulatory mutants like $c p h 1 \Delta / \operatorname{efg} 1 \Delta$. Interestingly, the presence of Tup1 was required for this positive effect.
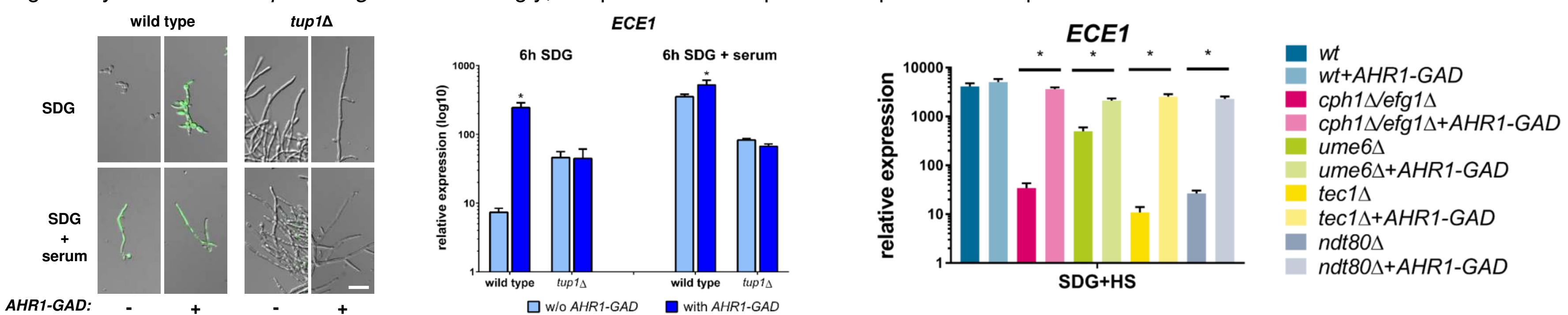

The indicated strains were grown in SDG+HS for $6 \mathrm{~h}$ at $37^{\circ} \mathrm{C}$ prior to microscopy or RNA isolation. Scale bar: $20 \mu \mathrm{m}$. Gene Expression was normalized as mentioned above.

\section{Mcm1 is involved in the regulation of ECE1}

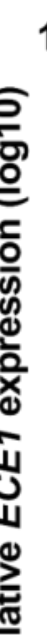

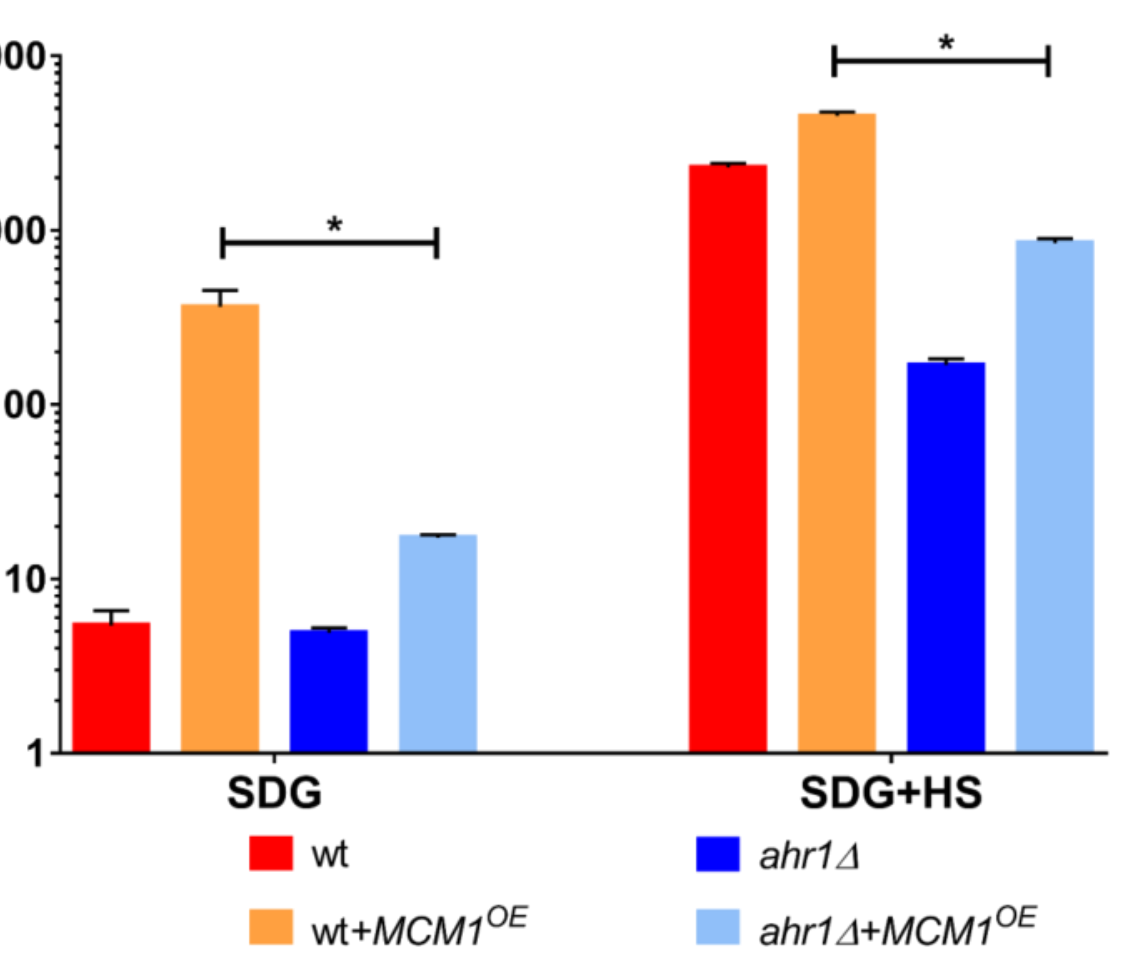

Mcm1 is a known interacting partner of Ahr1.

An overexpression of of this regulatory gene (MCM1 ${ }^{\mathrm{OE}}$ ) leads to increased ECE1 mRNA amounts.

However, this effect was significantly lower in ahr1 .
The transcriptional control of ECE1 is complex and includes multiple regulators. Our data showed that Ahr1 and Tup1 contribute to the activation of the gene, indicating that Tup1 is not solely a repressor. In addition, we were able to show that Mcm1 might be involved in the activation of ECE1. Further experiments will examine if these three regulators act together during the transcriptional control of ECE1.

\section{Acknowledgments}

We thank our collaboration partners B. Hube, J. Morschhäuser, I.D. Jacobsen and S. Vylkova for their support and contributions. This work was funded by the Deutsche Forschungsgemeinschaft (DFG) and the Bundesministerium für Bildung und Forschung (BMBF). 